\title{
Tratamiento adyuvante (Conpadri I) en osteosarcoma infantil (Valdivia 1980-1990)
}

\author{
Paola Zolezzi R.' ; Cecilia Faúndez V.' ; Jorge Linz. L. ${ }^{2}$; Roger Born M. ${ }^{3}$; \\ Pedro Valdjvia $\mathrm{C}^{4}{ }^{4}$, Jaime González G. ${ }^{4}$
}

\section{Chemotherapy after surgery in patients with osteosarcoma}

\begin{abstract}
In seven school age children, right jer-iur osteosarcomas were diagnosed at a Pediatric Hematologv and Oncoiogv Unit of Valdivia, Chile, from 1980 throughout 1986 and followed up until 1991. Their hystolcgical subtypeswere condroblasic in three patients: osteoblastic in two and telangiectatic in one child. Pulmonarv metastasis were not identified. One oatient rejected treatment. T'ne other six patients underwent right coxcfernora cisarticulation follows. ed by adjuxant cherrotherapy with Conpadri I schedule, which includes cyclophosphamide (CYT). Vyncristine ( $V C R$ ), doxorubycin (DAI), phenylalanine ni(rogen mustard (PAM). In three patients pulmonary Tetastasis were detected within the first three months of :his therapy and they died with in the same vear. Another three patients completed full chemotherapy. one of theri had a lung metastasis one month ater completion of therapy. he rejected surgica! resection and died 36 rronths after starting the treatment. The remaining two patients, both females, are stIlf alive and their disease free survivals (DFS) are 138 and 120 months respectivelv.
\end{abstract}

(Key words: osteosarcoma, chemotherapy.)

Los tumores malignos primarios del hueso constituyen sólo $2 \%$ de las neoplasias infantiles, siendo las más frecuentes el sarcoma de Ewing $y$ el osteosarcoma ${ }^{1}$. El osteosarcoma (OST) se presenta con una incidencia de $7 \mathrm{ca}$ sos nuevos por 1000000 de adolescentes/año ${ }^{2}$, considerándose que 80 a $90 \%$ de los pacientes tienen micrometástasis en el momento del diagnóstico inicial del tumor primario ${ }^{3}$. Con la am. putación o resección local del tumor se obtienen sobrevidas libre de enfermedad (SLE) a $\$$ años de 5 a $20 \%{ }^{3}$ que no se modifican mayormente al asociársele radioterapia (RT). El uso de qujmioterapia adyuvante ha permitido mejorar es. tos resultados. Con la aplicación del esquema Conpadri 1: ciclofosfamida, vincristina. doxorrubicina, mostaza nitrogenada de fenilalanina (melfalän), se ha observado una sobrevida total (ST) de $54 \%$ a 2 años plazo 4,5 . Sc comunican los resultados combinados de cirugía y quimioterapia adyuvante (Conpadri I) en los pacientes

1. Instituto de Hematologia, Facultad de Medicina. Universidad Austral de Chile.

2. Médico tratante, Consultorio Chonchi-Chiloé.

3. Servicio de Radiologia, Hospital Regional de Valdjvia.

4. Instituto de Traumatología, Facultad de Medicina. Universidad Austral de Chile. con OST menores de 15 años, provenientes de la X Region, estudiados entre enero 1980 y diciembre 1986, seguidos hasta junio 1991 .

\section{Material y Método}

Entre enero de 1980 y agosto de 1986 ingresarnn a la Unidad de Hematologia $y$ Oncologia Infantil del Hospital Regional de Valdivia siete niños provenientes de la X Región, con diagnóstico de osteosarcoma, basado en el estudio radiológico de la zona afectada seguido de biopsia quirúrgica o por punción y confirmación histológica.

El estudio de todos ellos incluyó hemograma. perfil bioquímico, examen de orina, radiografía de tórax, con planigrafía en los casos de sospecha de metástasis pulmonares, y clasificacjón del subtipo histológico de osteosarcoma según Schajovics.

El tratamiento consistió en cirugía, realizada entre 15 y 21 dias consecutivos a la biopsia diagnóstica, reemplazo de la extremidad afectada con una prótesis y kincsioterapia, seguidos por quimoterapia adyuvante, iniciada 14 días después de la cirugía, empleando doxorrubicina $(1,5 \mathrm{mg} \cdot \mathrm{kg}$ e.4., por una vez, dia 10 en las semanas $1,3,6,18,21,24 ; 1,0 \mathrm{mg} \cdot \mathrm{kg}$ e.v., por una vez, día 10 en las semanas $36,39,54,57$ ); vincristina $12 \mathrm{mg} \cdot \mathrm{m}^{2}$ e.v., por una vez, dia $1^{\circ}$ en las semanas $1,2,3,4,5,6 ; 1,5 \mathrm{mg} \cdot \mathrm{kg}$ e.v., por una vez, día $1^{\circ}$ las kemanas $12,13,30,31,42,43$ ) ciclofosfamida (10 $\mathrm{mg} \cdot \mathbf{k g} \cdot$ día por ? dias, en las semanas 12, $48,66)$ y melíalán $(0,3 \mathrm{mg} \cdot \mathrm{kg}$ oral, dosis única, en las semanas $30.42,60.72$ ). 
Se realizó control de hemograma y perfil bioquími co al inicio de cada cura, radiografía de tórax cada dos meses y electrocardiograma previo a la administración de doxorrubicina. Frente a la aparición de metástasis pulmonar única se planificó cirugía. Una vez finalizada la quirnioterapia, el control se realizó cada seis meses con hemograma, perfil bioquímico y radiografía de tórax.

\section{Resultados}

De los siete pacientes estudiados (cuatro varones), con edades entre 10 y 14 años 8 meses, cuatro provenían de la provincia de Valdivia, dos de Llanquihue y uno de Chiloé. Todos consultaron por aumento de volumen, dolor e impotencia funcional progresiva de una semana a tres meses de evolución, existiendo el antecedente de traumatismo previo en tres casos. En los siete niños la localización del tumor estaba en el tercio distal de fémur derecho y la radiología era altamente sugerente de proceso neoplásico.

Se efectuó biopsia quirúrgica en cinco casos y biopsia por punción en dos, correspondiendo a la variedad telangectásica en un caso, condro. blástica en tres, osteoblástica en dos y fibroblástica en otro.

En uno de los pacientes con osteosarcoma de tipo osteoblástico los padres rechazaron el tratamiento, efectuándose, por lo tanto, amputación subtrocantereana en dos pacientes $\mathrm{y}$ desarticulación de cadera en cuatro, confirmándose en todos ellos el subtipo histológico. Ante la pérdida de la extremidad afectada, dos pacien. tes de sexo masculino presentaron alteraciones anímicas agudas, recibiendo psicoterapia de apoyo. La aceptación de la prótesis, realizada en cinco, fue muy buena. En relación con la quimioterapia se observaron vómitos, depresiones moderadas de la serie blanca de rápida recuperación y alopecia. Un paciente sufrió varicela leve, y tres tuvieron infecciones dentarias de fácil tratamiento.

Tres pacientes completaron la quimioterapia, encontrándose dos de ellos vivos con una sobrevida libre de enfermedad de 138 meses y 120 meses, respectivamente. En el tercero (un niño con la variedad telangectásica) se detectó una metástasis pulmonar un mes después de suspender la quimioterapia, pero los padres rechazaron todo tratamiento, falleciendo el paciente a los 36 meses de hecho el diagnóstico. Las otros tres pacientes presentaron metástasis pulmonares múltiples dentro de los primeros 6 meses siguien. tes al diagnóstico y fallecieron, respectivamente, 10,9 y 8 meses después.

\section{Comentario}

Pese a lo reducido de la casu istica analizada, el protocolo aplicado, similar en todos los casos, con una mediana de sequimiento suficientemente prolongada, permite atribuir a la quimioterapia adyuvante, esquema Conpadri l, el resultado que consideramos favorable, de sobrevida libre de enfermedad de 33\%. Esta experiencia (1980-1986) nos ha proporcionado una base de referencia para comparar los resultados con nuevos esquemas terapéuticos ${ }^{6,}$, que demuestran mayor eficacia, entre ellos el protocolo Nacional Infantil para Osteosarcoma ${ }^{*}$.

La elección del esquema Conpadri I se debió a su conocida buena tolerancia y a su costo relativamente bajo, que es accesible a las disponibilidades financieras de nuestro medio hospitalario. El rápido crecimiento de este tipo de tumor maligno, la tardanza en consultar, en hacer el diagnóstico o la derivación a un centro que no cuenta con tomografía axial computadorizada, pueden haber repercutido negativamente en las expectativas de sobrevida de estos niños.

En la actualidad, la atención de los pacientes con osteosarcoma es hecha por equipos oncológicos multiprofesionales que en conjunto pueden caracterizar más completamente el tumor y su grado de extensión, empleando técnicas de reemplazo del hueso afectado, que permiten conservar la función del segmento comprometido y nuevos esquenas adyuvantes de quimioterapia que prolongan la sobrevida en un número cada vez mayor de pacientes.

\section{Resumen}

Siete escolares con osteosarcoma de fémur derecho, provenientes de la X Región, fueron atendidos en la unidad de Hematología y Oncología Pediátrica del Hospital de Valdivia, entre los años 1980 y 1986 y seguidos hasta 1991. Los subtipos histológicos encontrados fueron condroblästico en tres casos, osteoblástico en dos pacientes, fibroblástico en uno y telangectásico en uno. No se detectaron metástasis pulmonares en el momento de hacer el diagnóstico. Un paciente rechazó tratamiento, los seis restantes fueron tratados con desarticulación coxofemoral derecha seguida de quimioterapia adyuvante 
con el esquema Conpadri I: doxorrubicina, vincristina, ciclofosfamida y mostaza nitrogenada de fenilalanina (melfalán). Tres pacientes presentaron metástasis pulmonares dentro de los primeros meses después de iniciar el tratamiento y fallecieron antes de un año. Tres completaron el esquema de quimioterapia (extensión total 72 semanas). Uno de estos últimos presentó metástasis pulmonares al mes de suspendida la quimioterapia, rechazó la resección quirúrgica y murió 36 meses después. Las dos pacientes restantes se encuentran vivas, habiendo cumplido sobrevidas libre de enfermedad de 138 y 120 meses, respectivamente.

(Palabras clave: osteosarcoma, quimioterapia.)

\section{Referencias}

1. Jaffe $N$ : Malignant bone tumors. In Lanzkowsky P (ed.) "Pediatric Oncology". McGraw Hill Book Co., New York, 1983, pp. 293-309.
2. Badia Torrella: Sarcoma osteogénico. En "Oncología Pediátrica". Editorial Pediátrica. Barcelona. 1979. pp. 151-161.

3. Schaller $R$, Haos J. Schaller J, Morgan A, Bleyer $A$ : Improved survival in children with osteosarcoma following resection of pulmonary metastasis. J. Pediatr Surg 1982:17:516-550.

4. Herson $J$, Sutow $w$, Elder $K$ et al. Adyuvant chemotherapy in nonmetastatic osteosarcoma. Med Pediatr Oncol 1980; 8: 343-352.

5. Tokaue $Y$. Slopis J, Anzai T, Robertson $R_{+} J a f f e N$ : Succesful treatment of pulmonary and abdominal metastatic osteosatcoma. Med Pediatr Oncol 1985: 13: 126-128.

6. Makley J, Krailo $\boldsymbol{M}$, Ertel $I$ et al.: The relationship of various aspects of surgical management to outcome in childhood nonmetastatic osteosarcoma: A report from the childrens cancer study group. J Pediatr Surg 1988; 23: 146-151.

7. Meyers P: Malignant bone tumors in children: Osteosarcoma, Hematol Oncol Clin North Am 1987: I: 655-665.

8. Protocolos de Tratamiento Cánceres Infantiles. Programa Nacional de Drogas Antineoplásicas. Ministerio de Salud, 1987. 